\title{
ISOENZIMAS NA DIFERENCIAÇÃO DE SEMENTES DE TRÊS ESPÉCIES DO GÊNERO Euterpe ${ }^{1}$
}

\author{
Cibele Chalita Martins², Marilene Leão Alves Bovi ${ }^{3}$ Edson S. Mori² e João Nakagawa²
}

\begin{abstract}
RESUMO - A análise e o estudo das características morfológicas de sementes e plântulas de palmito-vermelho (Euterpe espiritosantensis Fernandes), juçara (E. edulis Mart.) e açaí (E. oleracea Mart.) não permitem que o analista de sementes, ou o melhorista, faça a identificação e a diferenciação inequívoca das espécies. Neste trabalho, buscou-se avaliar o potencial discriminante da técnica de eletroforese para sementes dessas espécies, utilizando-se nove sistemas enzimáticos. Foram realizadas análises de eletroforese de isoenzimas, testandose 30 embriões ( 0 a $1 \mathrm{~mm}$ de protrusão) de cada espécie, por corrida e por sistema enzimático. Para a identificação das bandas e determinação do perfil eletroforético foram realizadas, no mínimo, 30 corridas por sistema enzimático avaliado, em gel de poliacrilamida (7,5\%). Constatou-se que, dentre as isoenzimas testadas, a polifenol-oxidase e a fosfatase-ácida mostraram-se instáveis, raramente possibilitando a visualização das bandas. As isoenzimas $\alpha$ e $\beta$-esterase nem sempre possibilitaram o aparecimento de bandas visíveis, principalmente para E. espiritosantensis, mas foram capazes de distinguir E. edulis de E. oleracea. A glucose-6-fosfato desidrogenase e a glutamatodesidrogenase revelaram perfis eletroforéticos nítidos em todas as corridas, mas a posição das bandas não permitiu a diferenciação das três espécies estudadas. As isoenzimas mais eficientes na avaliação da pureza genética e na diferenciação das sementes foram fosfoglucomutase, fosfoglucose isomerase e peroxidase, por apresentarem perfis eletroforéticos distintos.
\end{abstract}

Palavras-chave: Euterpe espiritosantensis, Euterpe edulis, Euterpe oleracea e marcadores isoenzimáticos.

\section{ISOENZYMES IN THE DIFFERENTIATION OF THREE Euterpe SPECIES}

\begin{abstract}
The study and analysis of the morphological seed and seedlings characteristics of red-palmito (Euterpe espiritosantensis Fernandes), jussara (E. edulis Mart.) and assai (E. oleracea Mart.) do not allow the seed technologist, or even the plant breeder, to correctly distinguish the species. In this research the discriminator potential of the electrophoresis technique was evaluated for seeds of these species, using nine enzymatic systems. Thirty embryos ( $O$ to $1 \mathrm{~mm}$ of protrusion) of each species were used for enzymatic system and electrophoresis run. In order to identify the bands and the correspondent electrophoretic profile, at least 30 runs per enzymatic system were performed in study, using polyacrylamide gel (7.5\%). Among the tested isoenzymes, polyphenol oxidase and the acid phosphatase were unstable, without allowing bands visualization. The $\alpha$ and $\beta$-esterase isoenzymes could not always be visualized by clear bands, especially for $\boldsymbol{E}$. espiritosantensis, but both systems were able to distinguish between $\boldsymbol{E}$. edulis and $\boldsymbol{E}$. oleracea. The glucose-6-phosphate dehydrogenase and the glutamate dehydrogenase revealed well-marked electrophoretic profiles in all runs, however bands position did not allow the differentiation among the three species. The most efficient isoenzymes that can be used to evaluate genetic purity of seed lots and differentiate the seeds were phosphoglucomutase, phosphoglucose isomerase and peroxidase, by producing strong and distinctive electrophoretic profiles.
\end{abstract}

Keywords: Euterpe espiritosantensis, Euterpe edulis, Euterpe oleracea and allozyme markers.

\footnotetext{
${ }^{1}$ Recebido em 19.07.2006 e aceito para publicação em 30.10.2006.

${ }^{2}$ Departamento de Produção Vegetal, UNESP/FCA, Cx.P. 237, 18603-970 Botucatu, SP.

${ }^{3}$ Instituto Agronômico, cx.P. 28, 13001-970 Campinas, SP.
} 


\section{INTRODUÇÃO}

O palmito-vermelho (Euterpe espiritosantensis Fernandes) e a juçara (Euterpe edulis Mart.) são palmeiras nativas da Floresta Atlântica do Brasil. O primeiro é encontrado em formações florestais naturais na região de Santa Teresa, no Estado do Espírito Santo (FERNANDES, 1989), e em Ituberá e Itabuna, no Estado da Bahia (BOVI et al., 1987a; 1994). O segundo ocorre desde o Rio Grande do Sul até a Bahia e tem grande importância na composição florística de matas ciliares e na conservação de áreas perturbadas ou de mata secundária (BORÉM e OLIVEIRA-FILHO, 2002; MEIRA NETO et al., 2003). O açaizeiro (Euterpe oleracea Mart.) é palmeira nativa da região norte do Brasil, ocorrendo nos Estados do Pará, Amazonas, Maranhão e Amapá, estendendo-se ainda às Guianas e Venezuela (CALZAVARA, 1972; BOVI e CASTRO, 1993; GAMA et al., 2002).

Essas espécies vêm sendo exploradas de forma extrativista e predatória ao longo dos anos para a obtenção do palmito, produto valorizado nos mercados interno e externo. Antes da década de 1960, a produção básica de palmito vinha principalmente da costa meridional do país, sendo extraída da palmeira juçara. O Estado de São Paulo era, então, o principal produtor, e o ritmo de exploração, sem o correspondente replantio, reduziu rapidamente o número de palmeiras da região. Essa escassez de matéria-prima acarretou a mudança das maiores empresas processadoras de palmito para o Estado do Pará, então com extensas reservas de açaizeiros. Bovi (1998) considerou o Pará como sendo o principal Estado produtor, com cerca de $92 \%$ da produção brasileira, seguido por São Paulo, com apenas $4 \%$ da produção nacional.

A partir da década de 1970, plantas de açaí e de palmito-vermelho, este em menor escala, foram introduzidas nos estados do Sul e Sudeste do país, principalmente por instituições públicas, para pesquisa e ensino, e se propagaram. Foram implementados, inclusive, programas de melhoramento genético, com a produção de híbridos obtidos por cruzamentos controlados e, ou, naturais entre E. oleracea e outras espécies de Euterpe sem perfilhamento, entre elas $E$. edulis e E. espiritosantensis (BOVI, 1984; BOVI et al., $1987^{\mathrm{a}}$,b; BOVI e CASTRO, 1993; BOVI, 2004). Na década de 1990, aumentou a procura por sementes para o cultivo comercial e para a implantação de programas de manejo sustentável e, ou, recomposição de matas nativas.
A análise e estudo das características morfológicas de sementes e plântulas de E. espiritosantensis, E. edulis e E. oleracea até cinco meses após a semeadura não permitem que o analista de sementes, ou o melhorista, faça a identificação e diferenciação inequívoca das espécies apenas pelas características morfológicas (BOVI et al., 1987a,b; BALLVÉ, 1988). Características como coloração, tamanho e pilosidade, entre outras, variam em função das matrizes utilizadas, das condições de fertilidade do solo, e do clima do local de sua produção, da época de colheita e do período de armazenamento das sementes.

A identificação correta das diferentes espécies é de interesse científico e econômico. Identificações incorretas possibilitam misturas de espécies e variedades, por exemplo, na produção e comercialização de sementes. Isso resulta em prejuízos, por levar à utilização de técnicas inadequadas de cultivo, manejo e utilização, além do controle de espécies vegetais. Esse fato é de tal importância que a maioria dos países adota padrões legais de tolerância máxima de contaminação de lotes de sementes comerciais por sementes de outras espécies e variedades.

O potencial de técnicas eletroforéticas em sistemas de enzimas polimórficos na identificação de espécies e variedades de plantas tem sido largamente reconhecido, principalmente, em razão de se constituírem em marcadores estáveis, pouco influenciados pelo meioambiente (KELLER-GREIN et al., 1996). Os princípios dessas técnicas para a utilização na identificação genética de sementes foram detalhados por Payne (1987), e vários protocolos para análises eletroforéticas foram determinados (CHELIAK e PITEL, 1984; ALFENAS, 1998). A principal limitação do seu uso consiste da necessidade de se conhecer, em cada espécie ou grupo de espécies, os sistemas enzimáticos mais discriminantes. Uma vez conhecidos tais sistemas, essa técnica se torna relativamente simples e rápida.

Em palmiteiro, Ballvé (1988) iniciou os estudos de isoenzimas desenvolvendo um conjunto de metodologias de eletroforese em gel de amido, tendo analisado diversas introduções de E. edulis e E. oleracea e respectivos híbridos. Nesse trabalho, foram estudadas as isoenzimas de peroxidase, esterase, fosfatase ácida, fosfoglucose isomerase, álcool desidrogenase, enzima málica, polifenol oxidase e glutamato-oxaloacetato transaminase em tecidos de caule, palmito e pólen de 
plantas adultas, raiz de mudas e tecidos de folhas de plantas em diversas fases de desenvolvimento, verificando-se a possibilidade da utilização do polimorfismo enzimático na identificação de híbridos e caracterização da origem de plantas. Nas pesquisas seguintes, realizadas com Euterpe spp, utilizaram-se marcadores moleculares nessa mesma linha, ou seja, caracterização de populações naturais ou de bancos ativos de germoplasma quanto a cruzamentos, endogamia, parentesco, origem, identificação e variabilidade genética de populações (REIS, 1996; SAWAZAKI et al.,1998; SEBBENN e SEOANE, 2005). No entanto, essas técnicas também podem ser aplicadas para a avaliação da qualidade genética, fisiológica ou sanitária de sementes, e isso tem sido realizado com eficiência para várias espécies vegetais de importância econômica (CARVALHO et al., 2000).

Assim, este trabalho teve por objetivo avaliar o potencial da técnica de eletroforese de isoenzimas na diferenciação de sementes de palmito-vermelho, juçara e açaí.

\section{MATERIAL E MÉTODOS}

Frutos maduros de palmito-vermelho, juçara e açaí foram coletados manualmente, em 13.10.1998, 23.02.1999 e 01.04.1999, respectivamente, na Estação Experimental do Instituto Agronômico de Campinas (IAC), localizada

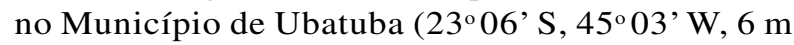
acima do nível do mar), Estado de São Paulo. A seguir, foram acondicionados em embalagem de polietileno e transportados até o Laboratório de Análise de Sementes do Departamento de Agricultura e Melhoramento Vegetal da Faculdade de Ciências Agronômicas da UNESPCampus de Botucatu, SP, onde foram despolpados através de fricção contra peneira de malha de aço, sob água corrente. Para o ensaio, utilizaram-se sementes provenientes de 17, 10 e 20 plantas-mãe, respectivamente, de E. espiritosantensis, E. edulis e E. oleracea.

As sementes foram postas para germinar em temperaturas alternadas de $20^{\circ} \mathrm{C}$ por 16 horas no escuro e $30^{\circ} \mathrm{C}$ por $8 \mathrm{~h}$ sob luz branca), sobre vermiculita esterilizada $\left(120^{\circ} \mathrm{C} / 12 \mathrm{~h}\right)$ e umedecida, dentro de caixas plásticas transparentes $(11 \times 11 \times 3,5 \mathrm{~cm})$. Botões germinativos recém-emitidos (após a abertura do opérculo, embriões com 0 a $1 \mathrm{~mm}$ de protrusão, três a sete dias após a semeadura) foram extraídos com o auxílio de um bisturi e submetidos à análise eletroforética. Nas análises de eletroforese de isoenzimas foram utilizados
30 embriões de cada espécie por tratamento, ou seja, seis embriões por célula, que foram congelados em nitrogênio líquido e macerados com um bastão de vidro com ponta polida em forma de cone, dentro de tubos plásticos contendo $0,5 \mathrm{~mL}$ de solução extratora. A solução extratora utilizada foi a número 1, prescrita em Alfenas (1998), que tem a seguinte composição química: $0,6 \mathrm{~g}$ de fosfato de sódio bibásico (0,034 M), $7 \mathrm{~g}$ de sacarose (0,2 M), 2,56 g de PVP-40 (2,56\%), 0,05 g de DTT (3 mM), 0,1 g de L-ácido ascórbico (5,7 mM), 0,1 g de DIECA (5,8 mM), 0,05 g de bisulfito de sódio (2,6 $\mathrm{mM}), 0,05 \mathrm{~g}$ de borato de sódio (2,5 mM), 0,2 mL de 2-mercaptoetanol (0,2\%), $1 \mathrm{~g}$ de polietilenoglicol-6000 (1\%) e $100 \mathrm{ml}$ de água deionizada (q.s.p.). O procedimento de extração foi realizado mantendose a base dos tubos com solução e embriões imersa em recipiente com água e gelo $\left(0^{\circ} \mathrm{C}\right)$, em sala com temperatura ambiente de $10^{\circ} \mathrm{C}$.

Os extratos obtidos foram centrifugados a 6.000 $\mathrm{G}$ por $10 \mathrm{~min}$, a $3^{\circ} \mathrm{C}$, e o sobrenadante, recentrifugado nas mesmas condições. Cada corrida foi realizada com amostras recém-extraídas ou armazenadas por até três dias a $-20^{\circ} \mathrm{C}$. Na corrida eletroforética, as alíquotas pipetadas foram padronizadas pelo teor de proteínas, utilizando-se $200 \mu \mathrm{g}$ de proteína por amostra, adicionandose $0,3 \mathrm{~mL}$ do corante azul de bromofenol na soluçãotampão do eletrodo, como marcador. As corridas foram realizadas pelo método de Warburg e Christian (1941), quantificando-se as proteínas com a seguinte fórmula: Concentração de Proteína $(u \mathrm{~g} / u \mathrm{~L})=\left(1,55 . \mathrm{D}_{280}-0,76\right.$. $\left.\mathrm{D}_{260}\right) / 1000$, em que $\mathrm{D}_{260}=$ leitura da absorbância da amostra a ser medida em comprimento de onda de 260 nm e $D_{280}=$ leitura da absorbância da amostra a ser medida em comprimento de onda de $280 \mathrm{~nm}$.

Para a eletroforese, utilizaram-se solução-tampão do eletrodo de TRIS-glicina ( $\mathrm{pH} 8,3$ ) e géis de poliacrilamida na concentração de 5\% para o gel concentrador e 7,5\% para o separador (DAVIS, 1964; ALFENAS, 1998). As corridas foram conduzidas utilizando-se cuba dupla para eletroforese vertical, dentro de câmara incubadora regulada à temperatura de $3{ }^{\circ} \mathrm{C}$. A corrente elétrica para a corrida eletroforética foi de $150 \mathrm{~V}$ e $20 \mathrm{~mA}$ até a marca do corante alcançar o gel separador; a partir desse ponto, foi de $220 \mathrm{~V}$ e $40 \mathrm{~mA}$ até a marca do corante aproximar-se do final do gel $(2 \mathrm{~h})$. Utilizou-se o gel na espessura de $0,9 \mathrm{~mm}$, largura de $7,5 \mathrm{~cm}$ e altura de $7,0 \mathrm{~cm}$ para o gel separador e de $0,5 \mathrm{~cm}$ de altura para o concentrador. Para a identificação das bandas e determinação do perfil eletroforético foram realizadas, no mínimo, 30 corridas por sistema enzimático avaliado.

R. Árvore, Viçosa-MG, v.31, n.1, p.51-57, 2007 
Foram comparados os graus de polimorfismo de nove sistemas enzimáticos: polifenol-oxidase (PPO), fosfatase-ácida (ACT), $\alpha$ e $\beta$-esterase ( $\alpha$ e $\beta$-EST), glucose-6-fosfato isomerase (G6PDH), glutamatodesidrogenase (GDH), fosfoglucomutase (PGM), fosfoglucose isomerase (PGI) e peroxidase (PO). O preparo das soluções para cada sistema enzimático e os procedimentos de coloração utilizados seguiram a metodologia descrita por Alfenas (1998).

Os géis foram fotodocumentados e a interpretação dos resultados, baseada na presença, nitidez, número e localização das bandas. Para a identificação das bandas nas repetições das corridas, para cada sistema isoenzimático testado foi calculado o coeficiente de mobilidade relativa (Rf), que representa a relação entre a distância de migração da isoenzima (I) e a distância de migração do corante (Fm), isto é, $\mathrm{Rf}=\mathrm{I} / \mathrm{Fm}$.

\section{RESULTADO E DISCUSSÃO}

Dos nove sistemas enzimáticos testados nas três espécies de Euterpe, a polifenol-oxidase (PPO) e a fosfatase-ácida (ACT) mostraram-se os mais instáveis, raramente possibilitando a visualização das bandas. Das 30 corridas realizadas com ACT, em apenas duas foi possível visualizar bandas. Das 16 corridas realizadas com PPO, apenas cinco apresentaram bandas, mas só visíveis através do equipamento de fotodocumentação, devido à intensidade luminosa. Esses perfis eletroforéticos desapareceram do gel após aproximadamente $16 \mathrm{~h} \mathrm{e}$ não permitiram a diferenciação das espécies. Assim, foi descartada a utilização da PPO e da ACT na diferenciação das sementes das espécies avaliadas.

Determinadas enzimas não estão presentes em todos os estádios de desenvolvimento do indivíduo, e por isso a expressão, resolução e estabilidade das bandas isoenzimáticas nos géis dependem do tipo e da idade do material vegetal do qual são obtidas as amostras. Ballvé (1988), em trabalho desenvolvido com E. edulis e E. oleracea, obteve para ACT: baixa resolução quando o tecido usado para extração foi o coleóptilo; padrões de bandas estáveis, semelhantes e de resolução satisfatória para caule, meristema e palmito; e ausência de atividade para pólen, no caso de E. oleracea. Sawazaki et al. (1998) também obtiveram resultados favoráveis com o uso de ACT, mas os extratos foram obtidos de folhas de mudas com 6 meses de idade ou, mesmo, de plantas adultas.

R. Árvore, Viçosa-MG, v.31, n.1, p.51-57, 2007
Os sistemas enzimáticos $\alpha$ e $\beta$-esterase ( $\alpha$ e $\beta$ EST) apresentaram desempenho médio, havendo a visualização de bandas em aproximadamente $80 \%$ das corridas para E. edulis e E. oleracea e $20 \%$ para $E$. espiritosantensis. Nesta última espécie, mesmo nas corridas que permitiram a visualização das bandas, em algumas delas os perfis apresentaram-se fracos e de difícil identificação, dificultando o estabelecimento de um perfil eletroforético característico dessa espécie.

Nas corridas em que foram detectadas bandas com $\alpha$-EST e $\beta$-EST, constatou-se que ambas as enzimas exibiram padrões similares de bandas tanto para $E$. oleracea quanto para E. edulis (Figura 1). Assim, utilizando-se $\alpha$ ou $\beta$-EST, observou-se que E. edulis apresenta as bandas 0,12 e 0,33 , que não estão presentes em E. oleracea (Figura 1), ou seja, essas bandas são discriminativas e podem ser utilizadas na identificação e diferenciação das sementes das duas espécies. O poder discriminativo das esterases para identificação entre E. oleracea, E. edulis e seus híbridos já havia sido relatado anteriormente por Sawazaki et al. (1998), utilizando folhas de plântulas dessas palmeiras.

As isoenzimas glucose-6-fosfato desidrogenase (G6PDH) e glutamato-desidrogenase (GDH) revelaram perfis eletroforéticos nítidos em todas as corridas, nas três espécies (Figuras 2 e 3); no entanto, a posição das bandas não permitiu a diferenciação das três espécies de Euterpe simultaneamente. Usando-se a G6PDH, foi possível diferenciar somente E. espiritosantensis das demais espécies, através das bandas de Rf 0,34; 0,42; e 0,50 (Figura 2). As demais espécies apresentaram perfis similares, com bandas de Rf 0,25; 0,32; e 0,37 para E. oleracea e de Rf 0,26; 0,31; e 0,35 para E. edulis. Usando-se a isoenzima GDH (Figura 3), foi possível diferenciar E. oleracea das demais espécies pela posição e maior número de bandas $(\operatorname{Rf} 0,02 ; 0,04 ; 0,09 ; 0,11$; 0,$13 ; 0,15$; e 0,36), enquanto E. espíritosantensis e $E$. edulis apresentaram perfil eletroforético idêntico e, portanto, indiferenciável, com duas bandas na mesma posição em ambas as espécies ( Rf 0,12 e 0,26).

Os sistemas enzimáticos fosfoglucomutase (PGM), fosfoglucose isomerase (PGI) e peroxidase (PO) também apresentaram perfis eletroforéticos nítidos em todas as corridas, sendo capazes de identificar e diferenciar as sementes de E. espiritosantensis, E. edulis e E. oleracea (Figuras 4, 5 e 6). 


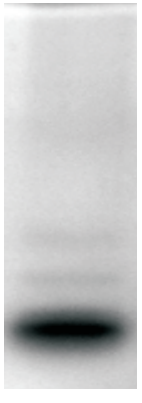

a

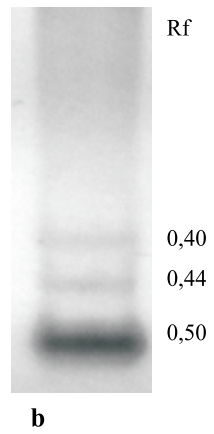

b

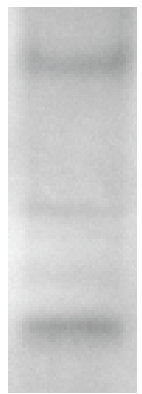

c d

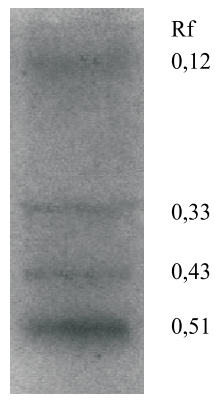

Figura 1 - Perfil eletroforético das isoenzimas a-esterase (a) e b-esterase (b) para embriões de Euterpe oleracea e de a-esterase (c) e b-esterase (d) para embriões de Euterpe edulis.

Figure 1 -Electrophoretic profile of-esterase (a) and besterase (b) isoenzymes in embryos of Euterpe olerace a and a-esterase (c) and b-esterase (d) in embryos of Euterpe edulis.

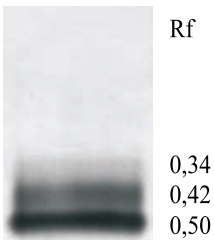

a

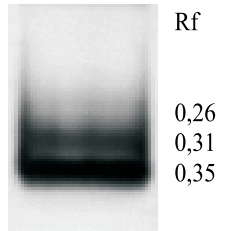

b

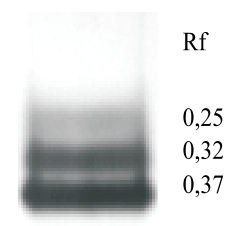

Figura 2 - Perfis eletroforéticos da isoenzima glucose-6fosfato desidrogenase (G6PDH) para embriões de Euterpe espiritosantensis (a), E. edulis (b) e E. oleracea (c).

Figure 2-Electrophoretic profiles of glucose-6-phosphate dehydrogenase isoenzyme (G6PDH) in embryos of Euterpe espiritosantensis (a), E. edulis (b) and E. oleracea $(c)$.

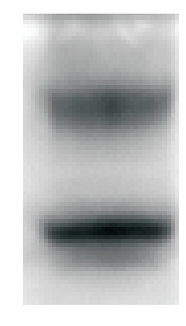

$\mathbf{a}$

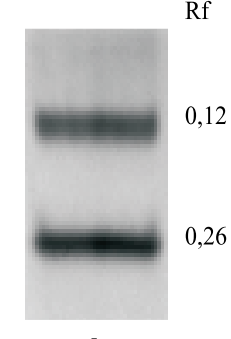

b

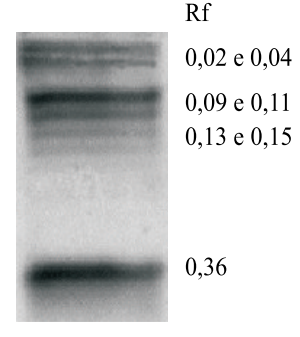

c
Figura 3 - Perfil eletroforético da isoenzima glutamatodesidrogenase (GDH ) para embriões de Euterpe espiritosantensis (a), E. edulis(b) e E. oleracea (c).

Figure 3-Electrophoretic profiles of glutamate dehydrogenase isoenzyme (GDH) in embryos of Euterpe espiritosantensis (a), E. edulis (b) and E. oleracea (c).
Observando o perfil eletroforético da PGM, constatouse que, para diferenciar as espécies, pode-se utilizar: o número de bandas, pois E. oleracea apresenta cinco delas, enquanto as demais, somente quatro; e a posição de bandas, posto que E. oleracea exibe bandas discriminativas nas posições 0,$04 ; 0,37$; e 0,61 , enquanto em $E$. edulis a banda discriminativa está na posição 0,42 e, em E. espiritosantensis, na posição 0,46 (Figura 4).

Utilizando o sistema enzimático da PGI, constatouse que, para diferenciar as espécies com base no perfil eletroforético, pode-se adotar o número de bandas, que são cinco, duas e quatro, respectivamente, para $E$. espiritosantensis, E. edulis e E. oleracea. Pode-se adotar, também, a posição de bandas, pois E. espiritosantensis apresenta bandas discriminativas de $\operatorname{Rf} 0,20 ; 0,23$; e 0,36 , E. edulis banda de Rf 0,33 e E. oleracea bandas de Rf 0,16; 0,26; e 0,40 (Figura 5).

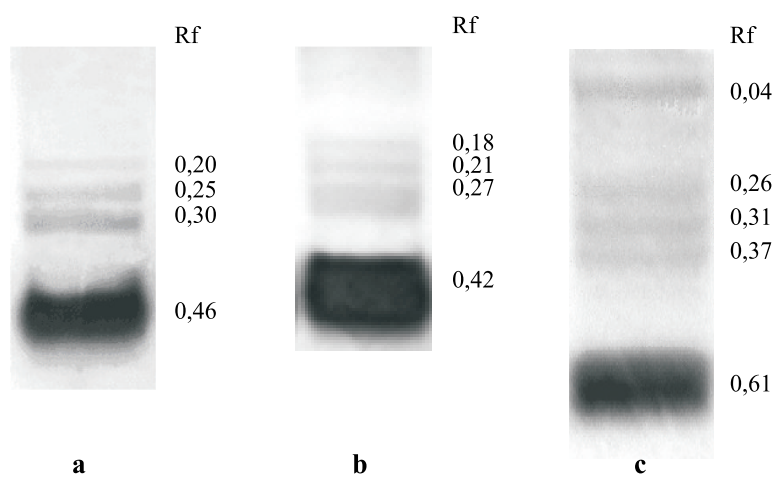

Figura 4 - Perfis eletroforéticos da isoenzima fosfoglucomutase (PGM) para embriões de Euterpe espiritosantensis (a) E. edulis (b) e E. oleracea (c).

Figure 4-Electrophoretic profiles of phosphoglucomutase isoenzyme (PGM) in embryos of Euterpe espiritosantensis (a), E. edulis (b) and E. oleracea (c).

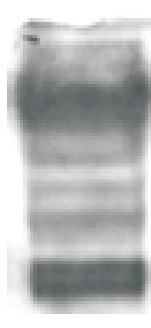

a

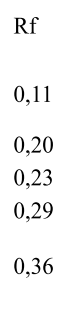

$\stackrel{\text { a }}{\text { Figura }} 5$ - Perfis eletroforéticos da isoenzima fosfoglucose

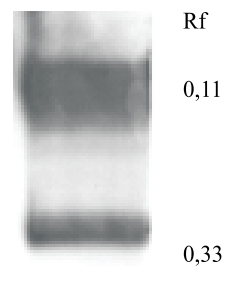

b

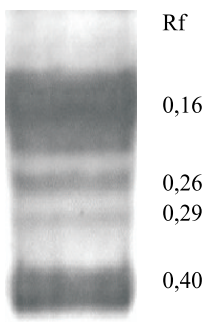
isomerase (PGI) para embriões de Euterpe espiritosantensis (a), E. edulis (b) e E. oleracea (c).

Figure 5-Electrophoretic profiles of phosphoglucose isomerase isoenzyme (PGI) in embryos of Euterpe espiritosantensis (a), E. edulis (b) and E. oleracea (c).

R. Árvore, Viçosa-MG, v.31, n.1, p.51-57, 2007 
Utilizando a PO, observou-se que as três espécies apresentam igual número de bandas (quatro), uma em posição mais inicial no gel e duas numa posição intermediária, próximas entre si, e a última numa posição mais final. Assim, a posição dessas bandas pode ser utilizada para discriminar as espécies. A posição da primeira banda só é capaz de diferenciar E. espiritosantensis ( $\mathrm{Rf} 0,05)$ das demais, pois E. edulis e E. oleracea apresentaram essa banda em posição similar (0,01 e 0,02, respectivamente). Porém, as duas bandas intermediárias e a banda final do gel podem ser usadas para diferenciar as três espécies, pois estão em posições distintas. Essas bandas exibem Rf de 0,$18 ; 0,27$; e 0,38 para E. espiritosantensis, 0,08; 0,15; e 0,22 para E. edulis e 0,13;0,17; e 0,29 para $E$. oleracea (Figura 6).

Boa definição e estabilidade para as enzimas G6PDH, PO, PGI e PGM também foram constatadas em trabalhos anteriores com E. edulis (REIS, 1998; SAWASAKI et al., 1998) e E. oleracea (SAWASAKI et al., 1998), mas a posição de algumas bandas e a presença de outras foram diferentes das identificadas neste trabalho. Tal comportamento pode ser explicado pelo fato de o material vegetal utilizado pelos autores, na extração, ser diferente e de idade mais avançada do que o usado no presente estudo: folhas de plantas com 6 meses de idade (SAWASAKI et al., 1998) e um 1 de idade ou adultas (REIS, 1998). Tanto o deslocamento uniforme das bandas quanto a ocorrência de maior ou menor número de bandas em amostras extraídas em diferentes estádios de desenvolvimento são fenômenos comuns e refletem modificações pós-tradução e, ou, expressão de loci específicas em certas fases de desenvolvimento dessas espécies de Euterpe (BALLVÉ, 1988).

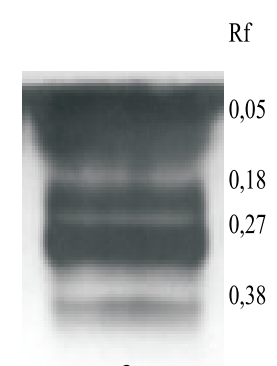

a

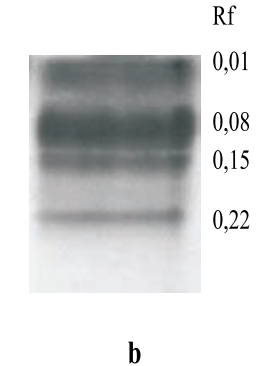

b

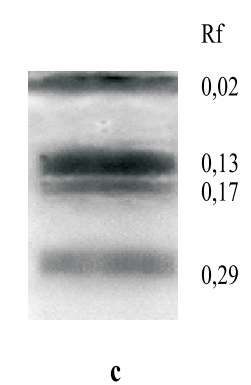

c
Figura 6 - Perfil eletroforético da isoenzima peroxidase (PO) para embriões de Euterpe espiritosantensis (a), E. edulis (b) e E. oleracea (c).

Figure 6-Electrophoretic profiles of peroxidase isoenzyme $(P O)$ in embryos of Euterpe espiritosantensis $(a)$, E. edulis (b) and E. oleracea (c).

R. Árvore, Viçosa-MG, v.31, n.1, p.51-57, 2007

\section{CONCLUSÃO}

Os perfis eletroforéticos de fosfoglucomutase (PGM), fosfoglucose isomerase (PGI) e peroxidase (PO) foram eficientes na avaliação da pureza genética e da diferenciação inequívoca de sementes de Euterpe espiritosantensis, E. edulis e E. oleracea.

\section{AGRADECIMENTOS}

À FAPESP (Programa Jovem Pesquisador) pelo financiamento desse trabalho.

\section{REFERÊNCIAS}

\section{ALFENAS, A.C. Eletroforese de isoenzimas e proteínas afins: fundamentos e aplicações em plantas e microorganismos. Viçosa, MG: Editora UFV,1998.574p.}

B ALLVÉ, R.M.L. Isoenzimas como marcadores genéticos em palmiteiro (Euterpe spp.). 1988. 86f. Dissertação (Mestrado em Biologia) - Universidade Estadual de Campinas, Campinas, 1988.

BORÉM, R.A.T.; OLIVEIRA FILHO, A.T. Fitossociologia do estrato arbóreo em uma toposeqüência alternada de mata atlântica, no município de Silva Jardim-RJ,Brasil. Revista Árvore, v.26, n.6, p.727-742, 2002.

BOVI, M.L.A. Resultados de pesquisa referentes à exploração, manejo e cultivo do açaizeiro. In: MOURÃ̃O, L.; JARDIM, M. A. G.; GROSSMANN, M. (Org.). Açaí - possibilidades e limites em processos de desenvolvimento sustentável no estuário amazônico. Belém: Editora Alves, 2004. p.53-78.

BOVI, M.L.A. Cultivo da palmeira real australiana visando a produção de palmito. Campinas: Instituto Agronômico, 1998. 26p. (Boletim, 172).

BOVI, M.L.A. Pesquisas em desenvolvimento com o açaizeiro no Instituto Agronômico, Estado de São Paulo. O Agronômico, v.36, n.2, p.155-178, 1984.

BOVI, M.L.A.; CASTRO, A. Assai. In: CLAY, J.W.; CLEMENT, C.R. (Ed.). Income generating forests and conservation in Amazonia. Rome: FAO, 1993. p.58-67. 
BOVI, M.L.A.; GODOY-JÚNIOR, G.; SAES, L.A. Híbridos interespecíficos de palmiteiro (Euterpe oleracea $\mathrm{x}$ Euterpe edulis). Bragantia, v.46, n.2, p.343-363, 1987a.

BOVI, M.L.A.; GODOY-JÚNIOR, G.; SAES, L.A. Pesquisas com os gêneros Euterpe e Bactris no Instituto Agronômico de Campinas. O Agronômico, v.39, n.2, p.129-174, 1987b.

BOVI, M.L.A. et al. Floral biology and reproductive system of Euterpe espiritosantensis Fernandes. Acta Horticulturae, v.360, n.8, p.41-56, 1994.

CALZAVARA, B.B.G. As possibilidades do açaizeiro no estuário amazônico. Belém: FCAP, 1972. 103p. (Boletim, 5).

CARVALHO, M. L.M.; VIEIRA, M. G.G.C.; VON PINHO, E. R.. Aplicação de técnicas moleculares no controle de qualidade das sementes.

Biotecnologia, Ciência \&

Desenvolvimento, n.17, p.44-47, 2000.

CHELIAK, W.M.; PITEL, J.A. Techniques for starch-gel electrophoresis of enzymes from forest tree species. Ontario: Patawawa National Foresty Institute, Canadian Foresty Service, 1984. 49p. (Information Report PI-X-42).

DAVIS, B.J. Disc electrophoresis; II. Method and application to human serum proteins. Annals of the New York Academy of Science, v.121, p.404-427, 1964.

FERNANDES, H.Q.B. Uma nova espécie de Euterpe (Palmae - Arecoideae - Arecaceae) do Brasil. Acta Botânica Brasilica, v.3, n.2, p.43-49, 1989,
GAMA, J. R. V.; BOTELHO, S. A.; BENTESGAMA, M. M. Composição florística e estrutura da regeneração natural de floresta secundária de várzea baixa no estuário amazônico. Revista Árvore, v.26, n.5, p.559-566, 2002.

KELLER-GREIN, G.; MAASS, B.; HANSON, J. Natural variation in Brachiaria and existing germplasm collection. In: MILES, J.W.; MAASS, B.L.; VALLE, C.B. (Ed.). Brachiaria: biology, agronomy and improvement. Cali: CIAT, 1996. p.16-35.

MEIRA NETO, J. A. A. et al. Origem, sucessão e estrutura de uma floresta de galeria periodicamente alagada em Viçosa-MG. Revista Árvore, v.27, n.4, p.561-574, 2003.

PAYNE, R.C. Seed and cultivar identification. Seed Science and Technology, v.15, n.3 p.641-644, 1987.

REIS, M.S. Distribuição e dinâmica da variação genética em populações naturais de palmiteiro (Euterpe edulis Mart.). 1996. 210f. Tese (Doutorado em Genética e Melhoramento de Plantas) - Escola Superior de Agricultura “Luiz de Queiroz”, Piracicaba, 1996.

SAWAZAKI, H.E. et al. Diversidade genética em palmeiras através de isoenzimas e RAPD.

Revista Brasileira de Biologia, v.58, n.4, p.681-691, 1998.

SEBBENN, A.M.; SEOANE, C. E.S. Estimativa de tamanho efetivo de endogamia por marcadores genéticos. Revista Árvore, v.29, n.1, p.1-8, 2005.

WARBURG, O.; CHRISTIAN, W. Biochemistry Zeithung, v.8, n.2, p.310-384, 1941. 
